\title{
A nonlocal discretization of fields
}

\author{
Rafael G. Campos and Eduardo S. Tututi \\ Escuela de Ciencias Físico-Matemáticas, \\ Universidad Michoacana \\ 58060 Morelia, Michoacán, México \\ E-mail: rcampos@zeus.umich.mx; tututi@zeus.umich.mx \\ L. O. Pimentel \\ Departamento de Física, \\ Universidad Autónoma Metropolitana-Iztapalapa \\ Apdo. Postal 55-534, México DF, 09340 México \\ E-mail: lopr@xanum.uam.mx
}

PACS: 11.15.Ha, 11.15.Tk, 02.60.Jh

Keywords: Nonlocal lattices, discrete differentiation, discrete fields.

\begin{abstract}
A nonlocal method to obtain discrete classical fields is presented. This technique relies on well-behaved matrix representations of the derivatives constructed on a non-equispaced lattice. The drawbacks of lattice theory like the fermion doubling or the breaking of chiral symmetry for the massless case, are absent in this method.
\end{abstract}




\section{Introduction}

The non-perturbative implementation of QCD uses the Feynman path-integral approach to yield a discretized version of the theory on a equispaced discrete space-time lattice where the main properties of the quantum fields are expected to be preserved [1]. However, properties such as chirality and uniqueness of lattice fields are not preserved. In fact, the Nielsen-Ninomiya theorem [2] states that for a local and translationally invariant hermitian discretization of the fields it is not possible to have simultaneously chiral symmetry and uniqueness (no doublers). Many attempts have been addressed to circumvent the restrictions of this no-go theorem (see for example [3]). Among these, one can find the use of higher number of dimensions [四, the construction of Dirac operators satisfying the Ginsparg-Wilson relation [5], the use of different lattice spacings for the gauge and the fermion fields [6], or the proposal of nonlocal actions (see for example [7] and references there in). The locality of the Dirac operator is in general a desired property, however, there are examples of operators that are topologically proper but not local [8]. In this letter we introduce another approach to the problem of discretization of fields. This is done by using a non-equispaced lattice, a projection of the partial derivatives that have been used to discretize the classical free Klein-Gordon and Dirac fields [9] and a discrete Fourier transform [10]. The classical discrete propagators approach to their continuum forms when the number of nodes tends to infinity and a relationship between this technique and that of path-integrals can be established. Such a technique can be seen as a projection of the quantum algebras on a finite linear space yielding matrix representations for the partial derivatives that produce hermitian actions with nonlocal kinetic terms [9, 11]. This scheme yields Dirac operators that are free from some drawbacks exhibited in the standard lattice method as the fermion doubling, the chiral symmetry breaking for the massless case or the yielding of different dispersion relations for bosons and fermions.

\section{Discrete formalism}

In order to be concise, we point out in this section the main features of the technique and leave the proofs of our assertions in the references [9, 10, 11, 12, 13].

We begin by choosing a non-equispaced lattice constructed with the set of $N_{\mu}(\mu=$ $0,1,2,3)$ zeros $x_{j}^{\mu}$ of the Hermite polynomial $H_{N_{\mu}}(\xi)$ along the direction $\mu$. This gives a total of $N=N_{0} N_{1} N_{2} N_{3}$ lattice points in a non-equispaced hypercubic structure. Now, we construct the four $N \times N$ matrices

$$
\begin{aligned}
& \mathbf{D}_{0}=D_{0} \otimes 1_{N_{3}} \otimes 1_{N_{2}} \otimes 1_{N_{1}}, \\
& \mathbf{D}_{3}=1_{N_{0}} \otimes D_{3} \otimes 1_{N_{2}} \otimes 1_{N_{1}}, \\
& \mathbf{D}_{2}=1_{N_{0}} \otimes 1_{N_{3}} \otimes D_{2} \otimes 1_{N_{1}}, \\
& \mathbf{D}_{1}=1_{N_{0}} \otimes 1_{N_{3}} \otimes 1_{N_{2}} \otimes D_{1},
\end{aligned}
$$


where $1_{N_{\mu}}$ is the identity matrix of dimension $N_{\mu}$ and $D_{\mu}$ is the skew-symmetric matrix

$$
\left(D_{\mu}\right)_{j k}= \begin{cases}0, & i=j, \\ \frac{1}{x_{j}^{\mu}-x_{k}^{\mu}}, & i \neq j .\end{cases}
$$

We also need

$$
\mathbf{S}=S_{0} \otimes S_{3} \otimes S_{2} \otimes S_{1}
$$

where $S_{\mu}$ is a diagonal matrix whose $j$ th nonzero element is given by the value of the function $\exp \left(-\xi^{2} / 2\right) H_{N_{\mu}}^{\prime}(\xi)$ at $\xi=x_{j}^{\mu}$.

Then,

$$
\mathrm{SD}_{\mu} \mathbf{S}^{-1}
$$

is a projection of the partial derivative $\partial_{\mu}$ in the subspace of functions $U$ generated by products of the form $u_{n}(\xi)=\exp \left(-\xi^{2} / 2\right) H_{n}(\xi)$ with $\xi=x^{\mu}, \mu=0,1,2,3$ and $n=$ $0,1, \ldots, N_{\mu}-1$. Note that $\left\{u_{n}(\xi)\right\}_{0}^{\infty}$, is a orthogonal basis for square integrable functions in one dimension. Thus, if $\psi(x) \in U$ and $\Psi$ denotes the $N \times 1$ vector of components

$$
\Psi_{q}=\psi\left(x_{q}\right) \equiv \psi\left(x_{j}^{1}, x_{k}^{2}, x_{l}^{3}, x_{m}^{0}\right),
$$

ordered according to

$$
q=j+(k-1) N_{1}+(l-1) N_{1} N_{2}+(m-1) N_{1} N_{2} N_{3}
$$

where $j=1,2 \ldots, N_{1}$ runs faster than $k=1,2 \ldots, N_{2}$, running faster than $l=1,2 \ldots, N_{3}$, running faster than $m=1,2 \ldots, N_{0}$, we have that the vector $\Psi_{, \mu}$ constructed with the values of $\psi_{, \mu}=\partial_{\mu} \psi$ at the site $x_{q}=\left(x_{m}^{0}, x_{l}^{1}, x_{k}^{2}, x_{j}^{1}\right)$ and ordered as in (3), is given by

$$
\Psi_{, \mu}=\mathbf{S D}_{\mu} \mathbf{S}^{-1} \Psi
$$

This means that (2) can be used to reproduce exactly the values of the partial derivatives of functions belonging to $U$ at the lattice sites. On the other side, if $\psi(x)$ is a square integrable function (considered as a function of just one variable $x^{\mu}$ ) that is not contained completely in $U$, Eq. (田) yields approximated values for the derivatives of $\psi(x)$ at the lattice points. The error can be estimated in very special cases [13] and become, as expected, related to the complement of $\psi(x)$ with respect to $U$. This behavior can be shown by considering a function of the form $\varphi(x)=a(x) \psi(x)$ with $\psi \in U$ (we will take just one variable in the following), and discretizing its derivative at $N$ nodes. Note that $\varphi$ is not necessarily contained in $U$ and in general, the vector $\Phi^{\prime}$ constructed with the values

$$
\varphi^{\prime}\left(x_{j}\right)=a\left(x_{j}\right) \psi^{\prime}\left(x_{j}\right)+a^{\prime}\left(x_{j}\right) \psi\left(x_{j}\right)
$$

is not given by $S D S^{-1} \Phi$, where $\Phi$ denotes the vector constructed with the values $\varphi\left(x_{j}\right)$. Thus, we address our attention to the residual vector $R=\Phi^{\prime}-S D S^{-1} \Phi$. Because of (5), $\Phi^{\prime}$ can be written as $A S D S^{-1} \Psi+A^{\prime} \Psi$ where $A$ is the diagonal matrix containing 
$a\left(x_{j}\right)$ along the main diagonal and $A^{\prime}$ the diagonal matrix containing $a^{\prime}\left(x_{j}\right)$. Thus, the remaining vector giving the error becomes

$$
R=\left(A S D S^{-1}-S D S^{-1} A+A^{\prime}\right) \Psi=S \Delta S^{-1} \Psi,
$$

where $\Delta$ is the matrix given by $\Delta=[A, D]+A^{\prime}$, i.e.,

$$
\Delta_{j k}= \begin{cases}a^{\prime}\left(x_{j}\right), & j=k, \\ \frac{a\left(x_{j}\right)-a\left(x_{k}\right)}{x_{j}-x_{k}}, & j \neq k .\end{cases}
$$

By rewriting the commutator as

$$
D A=A D+A^{\prime}-\Delta
$$

we can see the way in which the derivative of the product of functions $a(x) \psi(x)$ is handled by our method: just construct the diagonal matrix with nonzero elements $a\left(x_{j}\right)$ and apply the product $S D A S^{-1}$ to the vector $\Psi$. The error $R$ can be determined by considering the action of $\Delta$ on an arbitrary vector $V$. To this end, we take first the case where $a(x)$ is taken as the power $x^{n}$. It is not difficult to show that in such a case, $\Delta V$ is given by the linear combination

$$
\Delta V=b_{0} \xi_{n-1}+b_{1} \xi_{n-2}+\cdots+b_{n-2} \xi+b_{n-1},
$$

where $\xi_{l}$ is the vector whose $j$ th component is $x_{j}^{l}$ and the coefficients are given by $b_{l}=$ $\sum_{j=1}^{N} x_{j}^{l} V_{j}$. The same procedure to get (7) and the expansion of $a(x)$ in powers of $x$ yields again (7) for the partial sum $a_{n}(x)=\sum_{k=0}^{n} a_{n}^{k} x^{k}$, but the coefficients change to $b_{l}=\sum_{j=1}^{N} A_{l}\left(x_{j}\right) V_{j}$, where $A_{l}(x)$ is a polynomial of degree $l$. In any case, $R$ is always in the subspace spanned by ([) and determined by the higher power of $x$ in $a_{n}(x)$. Now, let us consider the part of $R$ given by $\Delta V$, where $V=S^{-1} \Psi$. Since $\psi(x) \in U$, the components of $\Psi$ are of the form $\Psi_{j}=\exp \left(-x_{j}^{2} / 2\right) f\left(x_{j}\right)$ (where $f(x)$ is a polynomial of fixed degree), the coefficients of (7) becomes

$$
b_{l}=\sum_{j=1}^{N} A_{l}\left(x_{j}\right) \frac{f\left(x_{j}\right)}{H_{N}^{\prime}\left(x_{j}\right)}, \quad l=0,1, \cdots, n-1,
$$

where $n$ is the higher power of $a_{n}(x)$. Therefore, $S \Delta S^{-1}$ projects $\Psi$ on the subspace spanned by the independent vectors constructed with the values of $\exp \left(-x^{2} / 2\right) x^{N+l-1}$ at the nodes. Thus, the error in the discretized derivative of a function $a(x) \psi(x)$ not belonging completely to $U$ depends on the components $u_{l}(x)=\exp \left(-x^{2} / 2\right) H_{l}(x)$ defined by the orthogonal complement of $U$. Now, to show the condition under which $b_{l}=0$, let us take the higher power of $A_{l}(x)$ in (8). This gives a sum of the form

$$
\sum_{j=1}^{N} \frac{x_{j}^{l} f\left(x_{j}\right)}{H_{N}^{\prime}\left(x_{j}\right)}
$$


By using the matrix representation of $d / d x$ given in [14] applied to the vector formed with the values of the function $g(x)=\left(x-x_{j}\right)^{l+1} f(x)$ at the nodes, one can show that if $f(x)$ is a polynomial of degree at most $N-l-2$, the sum given in (9) is zero for a power of $x_{j}$ less or equal to $l$. Thus, the derivative of the product $a_{n}(x) \psi(x)$ can be represented exactly by our discrete scheme whenever $\psi(x)$ is restricted to be in the subspace generated by the basis $u_{l}(x), l=0,1, \cdots, N-n-1$. In the case of four variables, $a(x)$ can be expanded in terms of the basis $u_{q}(x)=u_{m}\left(x^{0}\right) u_{l}\left(x^{3}\right) u_{k}\left(x^{2}\right) u_{j}\left(x^{1}\right)$, where $u_{n}(\xi)=\exp \left(-\xi^{2} / 2\right) H_{n}(\xi)$ and (6) becomes

$$
\mathbf{D}_{\mu} \mathbf{a}=\mathbf{a D}_{\mu}+\mathbf{a}_{, \mu}-\Delta_{\mu}
$$

where the projection matrix $\boldsymbol{\Delta}_{\mu}$ can also be given in terms of the one-dimensional matrix $\Delta_{\mu}$ through a similar relation to (1). Such a matrix makes a projection on the orthogonal subspace to the one generated by the basis $u_{q}(x)$. Therefore, we have a convergent discretization scheme if $N_{\mu}$ is large enough.

Let us take a look on the similarity transformation of (目) when $N_{\mu} \rightarrow \infty$. First of all, note that (14) implies that $\mathbf{D}_{\mu}$ can be considered as a representation of $\partial_{\mu}$ for tranformed vectors $\Phi$ of the form $\Phi=\mathbf{S}^{-1} \Psi$. Since

$$
H_{N_{\mu}}^{\prime}\left(x_{j}^{\mu}\right) \approx(-1)^{N_{\mu}+j} \frac{2 N_{\mu} !}{\Gamma\left(\frac{N_{\mu}+1}{2}\right)} \exp \left[\left(x_{j}^{\mu}\right)^{2} / 2\right], \quad N_{\mu} \rightarrow \infty,
$$

the $j$ th nonzero element of $S_{\mu}$ takes the asymptotic form $(-1)^{N_{\mu}+j} 2 N_{\mu}$ ! $\left./ \Gamma\left[\left(N_{\mu}+1\right) / 2\right)\right]$, therefore, the $j k$ element of $S_{\mu} D_{\mu} S_{\mu}^{-1}$ differs from the $j k$ element of $D_{\mu}$ only by $(-1)^{j+k}$. This means that in the limit $N_{\mu} \rightarrow \infty$, the transformed vector $\Phi=\mathbf{S}^{-1} \Psi$ differs from $\Psi$ only by an alternating change of sign along the $\mu$-direction. In other words, if we take into account the fact that the similarity transformation, given by $\mathbf{S}$, transforms $\Psi$ into itself, except for an alternating change of sign along each direction in the asymptotic limit, we always may use $\mathbf{D}_{\mu}$ instead (2) as representation of $\partial_{\mu}$.

The commuting matrices (1) can be diagonalized simultaneously by the unitary and symmetric matrix

$$
\mathbf{F}=F_{0}^{*} \otimes F_{3} \otimes F_{2} \otimes F_{1},
$$

(* means complex conjugate) where

$$
\left(F_{\mu}\right)_{j k}=\sum_{l=0}^{N_{\mu}-1}(i)^{l} \varphi_{l}\left(x_{j}^{\mu}\right) \varphi_{l}\left(p_{k}^{\mu}\right)
$$

and

$$
\varphi_{l}(\xi)=\sqrt{\frac{\left(N_{\mu}-1\right) ! 2^{N_{\mu}-1-l}}{N_{\mu} l !}} \frac{H_{l}(\xi)}{H_{N_{\mu}-1}(\xi)} .
$$

Here, $p_{j}^{\mu}$ is also a root of $H_{N_{\mu}}(\xi)$ and it represents an eigenvalue of the discretized linear momentum. It is possible, on physical grounds, to construct (12) as a convergent discrete Fourier transform [10]. Denoting by $[\cdots]_{k}$ the $k$ th column of a matrix, we have that

$$
\left[F_{\mu}\right]_{k} \rightarrow(-1)^{N_{\mu}+1}\left[F_{\mu}^{\dagger}\right]_{k},
$$


as $p_{k}^{\mu} \rightarrow-p_{k}^{\mu}$. Besides $\mathbf{F}^{\dagger}=\mathbf{F}^{-1}$, the basic properties of the four-dimensional discrete Fourier transform (11), are

$$
i \mathbf{D}_{0} \mathbf{F}=\mathbf{F P}^{0}, \quad-i \mathbf{D}_{j} \mathbf{F}=\mathbf{F P}^{j}
$$

and

$$
\lim _{N \rightarrow \infty} \mathbf{F}_{q q^{\prime}}=C_{N} e^{-i p_{q} \cdot x_{q^{\prime}}}
$$

where $C_{N}$ is the product of constants of the form円 $C_{N_{\mu}}=2^{N_{\mu}-3 / 2}\left(\Gamma\left[\left(N_{\mu}+1\right) / 2\right]\right)^{2} / N_{\mu}$ !, and $p_{q} \cdot x_{q^{\prime}}=p_{m}^{0} x_{m^{\prime}}^{0}-p_{l}^{3} x_{l^{\prime}}^{3}-p_{k}^{2} x_{k^{\prime}}^{2}-p_{j}^{1} x_{j^{\prime}}^{1}$. The order between indexes is given by (3). In (14) $\mathbf{P}^{\mu}$ is given by tensor products of the form (11) in terms of the diagonal matrix $P^{\mu}$ containing the eigenvalues $p_{j}^{\mu}$ along the main diagonal.

\section{Discrete Lagrangians}

The path-integral quantization of scalar and spinor fields uses the classical Lagrangian of the corresponding field. Thus, our aim in this section is to give the rules to obtain a discretized version of such a Lagrangian and their properties. In order to do this, let us take for instance the Lagrangian density for QED:

$$
L=\bar{\psi}\left(i \not \partial-e \gamma^{\mu} A_{\mu}-m\right) \psi-\frac{1}{4}\left(\partial_{\mu} A_{\nu}-\partial_{\nu} A_{\mu}\right)^{2}
$$

where $\psi=\psi(x)$ and $A_{\mu}=A_{\mu}(x)$ are the spinor and gauge fields respectively.

To get a discrete form of this Lagrangian we choose the four-dimensional lattice $x_{q}$ of the previous section, i.e, $N_{\mu}$ zeros of $H_{N_{\mu}}(\xi)$ as lattice points for each direction $\mu=0,1,2,3$ giving thus a total of $N=N_{0} N_{1} N_{2} N_{3}$ nodes. To maintain the relationship between the continuous and discrete field variables, let us denote by $\Psi$ the spinor vector of components $\Psi_{a q}=\Psi_{a}\left(x_{q}\right)$, where $a=1,2,3,4$ is the spinor index and $q$ is the lattice index ordered as in (3). A component of this vector can be described by only one index $r$ according to the order:

$$
r=j+(k-1) N_{1}+(l-1) N_{1} N_{2}+(m-1) N_{1} N_{2} N_{3}+(i-1) N_{1} N_{2} N_{3} N_{0}
$$

where the slower index is $i=1,2,3,4$. Thus, $\Psi$ is a vector of dimension $4 N$. The gauge vector, denoted by $\mathcal{A}_{\mu}$, has the components $\mathcal{A}_{\mu q}=\mathcal{A}_{\mu}\left(x_{q}\right)$ ordered as in (3), yielding a vector of dimension $N$. The values of the derivative $\partial_{\mu} A_{\nu}$ at the nodes are denoted by $\left(\mathcal{A}_{\nu, \mu}\right)_{q}$. Therefore, a discretization of (16) is

$$
\mathcal{L}_{q}=i \bar{\Psi}_{a q} \gamma_{a b}^{\mu}\left(\Psi_{, \mu}\right)_{b q}-\bar{\Psi}_{a q}\left(e \gamma_{a b}^{\mu} \mathcal{A}_{\mu q}+m \delta_{a b}\right) \Psi_{b q}-\frac{1}{4}\left[\left(\mathcal{A}_{\nu, \mu}\right)_{q}-\left(\mathcal{A}_{\mu, \nu}\right)_{q}\right]^{2}
$$

\footnotetext{
${ }^{1}$ In fact, there is a factor $(-1)^{j+k}$ in the asymptotic form of $\left(F_{\mu}\right)_{j k}$ that should be taken into account in $C_{N_{\mu}}$. However, this factor can also be treated along the same lines as in the previous paragraph.
} 
where $\bar{\Psi}_{a q}=\Psi_{b q}^{\dagger} \gamma_{b a}^{0}$. According to our procedure, the derivatives $\left(\Psi_{, \mu}\right)_{b q}$ and $\left(\mathcal{A}_{\nu, \mu}\right)_{q}$ can be approximated by $\left(\mathbf{D}_{\mu}\right)_{q r} \Psi_{b r}$ and $\left(\mathbf{D}_{\mu}\right)_{q r} \mathcal{A}_{\nu r}$, respectively $\left[\mathbf{D}_{\mu}\right.$ is used instead of (2) ]. Here, $a, b=1,2,3,4$ and $q, r=1, \cdots, N$. Repeated indexes are summed excepting $q$. When (18) is written in matrix form, the reference to the index $q$ disappears and the equation becomes the inner product

$$
\mathcal{S}=\bar{\Psi}\left[i \gamma^{\mu} \otimes\left(\mathbf{D}_{\mu}+i e \tilde{\mathcal{A}_{\mu}}\right)-m \mathbf{1}_{4 N}\right] \Psi-\frac{1}{4}\left(\mathbf{D}^{\mu} \mathcal{A}^{\nu}-\mathbf{D}^{\nu} \mathcal{A}^{\mu}\right)^{T}\left(\mathbf{D}_{\mu} \mathcal{A}_{\nu}-\mathbf{D}_{\nu} \mathcal{A}_{\mu}\right)
$$

where $\tilde{\mathcal{A}}_{\mu}$ is a diagonal matrix with the values $\mathcal{A}_{\mu q}$ along the main diagonal, $\bar{\Psi}$ is the transposed conjugate vector

$$
\bar{\Psi}=\left[\left(\gamma^{0} \otimes \mathbf{1}_{N}\right) \Psi\right]^{\dagger}=\Psi^{\dagger}\left(\gamma^{0} \otimes \mathbf{1}_{N}\right)
$$

$\mathbf{1}_{n}$ is the identity matrix of dimension $n$ and the superscript $T$ means transpose. Because (19) involves the sum over all $q$ and this means to sum over the spatial and temporal indexes, $\mathcal{S}$ is a discretization of the classical action save for a constant which gives the correct dimension of the action. Since $\mathbf{D}_{\mu}$ is real and skew-symmetric, $\mathcal{S}$ is real. By making the definitions

$$
\tilde{\mathbf{D}}_{\mu}=\mathbf{D}_{\mu}+i e \tilde{\mathcal{A}}_{\mu}
$$

and

$$
\mathcal{S}_{M}=-\frac{1}{4}\left(\mathbf{D}^{\mu} \mathcal{A}^{\nu}-\mathbf{D}^{\nu} \mathcal{A}^{\mu}\right)^{T}\left(\mathbf{D}_{\mu} \mathcal{A}_{\nu}-\mathbf{D}_{\nu} \mathcal{A}_{\mu}\right),
$$

$\mathcal{S}$ can be written in a simple form

$$
\mathcal{S}=\bar{\Psi}\left(i \tilde{\mathbf{D}}-m \mathbf{1}_{4 N}\right) \Psi+\mathcal{S}_{M},
$$

with the understanding that the slash notation involves tensor products in the discrete case.

\subsection{Chiral symmetry and absence of fermion doubling}

As we mentioned it, our discretization scheme presents some desirable features to be considered in studies of field theories such as QCD. First of all we want to show that the doublers are absent in this scheme. Let us begin with the free Dirac equation,

$$
(i \not \partial-m) \psi=0 \text {. }
$$

According to our method, the discretized version of this equation is

$$
\left(i \gamma^{\mu} \otimes \mathbf{D}_{\mu}-m \mathbf{1}_{4 N}\right) \Psi=0 .
$$

\footnotetext{
${ }^{2}$ Indeed, we are using dimensionless quantities for both the sites at the lattice (space-time and momenta) and the discrete fields.
} 
This is an eigenvalue equation for $m$ and it can be solved by using the discrete Fourier transform $\mathbf{F}$ given by (11). Denoting by $\mathbf{M}$ the diagonal matrix containing the eigenvalues $m_{q}$, (23) becomes

$$
\left(i \gamma^{\mu} \otimes \mathbf{D}_{\mu}\right) \Psi=\Psi \mathbf{M}
$$

where $\boldsymbol{\Psi}$ is the matrix whose $q$ th column corresponds to the eigenvector $\Psi_{q}$. Multiplying this equation by $\mathbf{1}_{4} \otimes \mathbf{F}^{\dagger}$ and using Eqs. (14) we obtain the transformed equation

$$
\left(i \gamma^{\mu} \otimes \mathbf{F}^{\dagger} \mathbf{D}_{\mu} \mathbf{F}\right) \mathbf{\Phi}=\left(\gamma^{\mu} \otimes \mathbf{P}_{\mu}\right) \mathbf{\Phi}=\mathbf{\Phi} \mathbf{M}
$$

where

$$
\boldsymbol{\Phi}=\left(\mathbf{1}_{4} \otimes \mathbf{F}^{\dagger}\right) \Psi
$$

is the matrix of transformed eigenvectors and $\mathbf{P}_{\mu}=g_{\mu \nu} \mathbf{P}^{\nu}$. This equation, the discrete version of $\not p \psi=m \psi$, says that $\mathbf{M}$ is similar to the matrix $\mathbf{P}$ defined by

$$
\mathbf{P}=\gamma^{\mu} \otimes \mathbf{P}_{\mu}
$$

By applying $\mathbf{P}$ again to (24), one can shows that $\mathbf{M}^{2}$ becomes diagonal and equal to $\mathbf{1}_{4} \otimes \mathbf{m}^{2}$, where

$$
\mathbf{m}^{2}=\left(\mathbf{P}^{0}\right)^{2}-\left(\mathbf{P}^{1}\right)^{2}-\left(\mathbf{P}^{2}\right)^{2}-\left(\mathbf{P}^{3}\right)^{2}
$$

Thus, the square of a mass eigenvalue, say $m_{r}$, is given by the discrete form of the energymomentum relation

$$
m_{r}^{2}=\left(p_{m}^{0}\right)^{2}-\left(p_{j}^{1}\right)^{2}-\left(p_{k}^{2}\right)^{2}-\left(p_{l}^{3}\right)^{2},
$$

where the indexes are ordered according to (17). Therefore, the doublers are absent.

Let us note that (26) yields a dense set of points $m_{r}^{2}$ in the set of real numbers since $p_{n}^{\mu}$ are zeros of the Hermite polynomial $H_{N_{\mu}}(\xi)$.

In the case where there is a gauge field coupled to a spinor field, the discretized Dirac equation is

$$
i \tilde{\mathbf{D}} \Psi=m \mathbf{1}_{4 N} \Psi .
$$

This equation defines the Dirac operator as $i \tilde{\mathbf{D}}$. Due to the properties of tensor products, this matrix satisfies trivially the discretized condition for chiral symmetry:

$$
\left(\gamma^{5} \otimes \mathbf{1}_{N}\right)(i \tilde{\mathbf{D}})+(i \tilde{\mathbf{D}})\left(\gamma^{5} \otimes \mathbf{1}_{N}\right)=0
$$

\subsection{Plane-wave solutions for the free Dirac field}

We can obtain solutions of the free equation (23) by anti-transforming (25). Let $\Phi_{q}$ be the eigenvector corresponding to $m_{q}$ (a vector of zeros everywhere save for the $q$ th element which is one). Since $m_{q}$ is degenerate, i.e., there are several values of $p_{q}^{\mu}$ giving the same 
eigenvalue $m_{r}^{2}$, the solution of (23) $\Psi_{m_{r}}$ corresponding to the mass eigenvalue $m_{r}$ is given by the linear combination

$$
\Psi_{m_{r}}=\sum_{p_{q}^{2}=m_{r}^{2}} \mathbf{c}_{q} \otimes[\mathbf{F}]_{q},
$$

where $p_{q}^{2}=\left(p^{\mu}\right)_{q}\left(p_{\mu}\right)_{q}$ and $\mathbf{c}_{q}$ is $1 \times 4$ vector. According to the asymptotic form of the elements of $[\mathbf{F}]_{q}$ given in (15), (27) corresponds to a plane-wave discretized solution of (22). Since (26) gives the same value $m_{r}^{2}$ under the interchange $p_{q}^{\mu} \rightarrow-p_{q}^{\mu}$ and $[\mathbf{F}]_{q}$ changes to $\left[\mathbf{F}^{\dagger}\right]_{q}$, except for a minus sign $[\mathrm{cf}$. (13)] that can be included in the linear combination, Eq. (27) splits in two sums, one corresponding to positive values of $p_{m}^{0}$ and the other to negative values, i.e.,

$$
\Psi_{m_{r}}=\sum_{p_{q}^{2}=m_{r}^{2}}^{+} \mathbf{a}_{q} \otimes[\mathbf{F}]_{q}+\sum_{p_{q}^{2}=m_{r}^{2}}^{-} \mathbf{b}_{q} \otimes\left[\mathbf{F}^{\dagger}\right]_{q}
$$

where the $+(-)$ superscript means to sum over points lying on the upper (lower) hyperboloids defined through

$$
p_{m}^{0}= \pm\left[m_{r}^{2}+\left(p_{j}^{1}\right)^{2}+\left(p_{k}^{2}\right)^{2}+\left(p_{l}^{3}\right)^{2}\right]^{1 / 2} .
$$

To find out the explicit forms of $\mathbf{a}_{q}$ and $\mathbf{b}_{q}$, let us take just one summand of each sum of (28) and define them as

$$
\Psi_{q}^{+}=\mathbf{a}_{q} \otimes[\mathbf{F}]_{q}, \quad \Psi_{q}^{-}=\mathbf{b}_{q} \otimes\left[\mathbf{F}^{\dagger}\right]_{q} .
$$

By applying (23) to $\Psi_{q}^{ \pm}$and taking into account that $\mathbf{D}_{\mu}[\mathbf{F}]_{q}=-i p_{\mu q}[\mathbf{F}]_{q}$ and $\mathbf{D}_{\mu}\left[\mathbf{F}^{\dagger}\right]_{q}=$ $i p_{\mu q}\left[\mathbf{F}^{\dagger}\right]_{q}$, where $p_{\mu q}$ is the $q$ th nonzero element of the diagonal matrix $\mathbf{P}_{\mu}$, we obtain that

$$
\left(\gamma^{\mu} p_{\mu q}-m_{q} \mathbf{1}_{4}\right) \mathbf{a}_{q}=0, \quad\left(\gamma^{\mu} p_{\mu q}+m_{q} \mathbf{1}_{4}\right) \mathbf{b}_{q}=0
$$

which are the equations for Dirac spinors evaluated at $p_{\mu q}$ and $m_{q}$ (an expected result since the discretization scheme presented in this letter is not involved with the spinor space). Therefore, the standard procedure to find the solutions of (30) can be followed step-by-step yielding the expansion of the wave function of a particle of mass $m_{r}$ in terms of plane waves

$$
\Psi_{m_{r}}=\sum_{p_{q}^{2}=m_{r}^{2}}^{+}\left[\left(m_{r} / p_{m}^{0}\right) \sum_{l=1,2} a_{q}^{l} \mathbf{u}_{q}^{l}\right] \otimes[\mathbf{F}]_{q}+\sum_{p_{q}^{2}=m_{r}^{2}}^{-}\left[\left(m_{r} / p_{m}^{0}\right) \sum_{l=1,2} b_{q}^{l} \mathbf{v}_{q}^{l}\right] \otimes\left[\mathbf{F}^{\dagger}\right]_{q},
$$

where $a_{q}^{l}$ and $b_{q}^{l}$ are complex numbers and

$$
\begin{aligned}
\mathbf{u}_{q}^{1} & =C_{m r}\left[1,0, p_{l}^{3} /\left(p_{m}^{0}+m_{r}\right), p_{j k}^{+} /\left(p_{m}^{0}+m_{r}\right)\right]^{T}, \\
\mathbf{u}_{q}^{2} & =C_{m r}\left[0,1, p_{j k}^{-} /\left(p_{m}^{0}+m_{r}\right),-p_{l}^{3} /\left(p_{m}^{0}+m_{r}\right)\right]^{T}, \\
\mathbf{v}_{q}^{1} & =C_{m r}\left[p_{l}^{3} /\left(p_{m}^{0}+m_{r}\right), p_{j k}^{+} /\left(p_{m}^{0}+m_{r}\right), 1,0\right]^{T}, \\
\mathbf{v}_{q}^{2} & =C_{m r}\left[p_{j k}^{-} /\left(p_{m}^{0}+m_{r}\right),-p_{l}^{3} /\left(p_{m}^{0}+m_{r}\right), 0,1\right]^{T} .
\end{aligned}
$$

Here, $C_{m r}=\left[\left(p_{m}^{0}+m_{r}\right) / 2 m_{r}\right]^{1 / 2}, p_{j k}^{ \pm}=p_{j}^{1} \pm i p_{k}^{2}$ and $j, k, l, m$ are the indexes of the components of $p_{q}^{2}$ satisfying (29) for the given eigenvalue $m_{r}$. 


\subsection{Gauge invariance and the covariant derivative}

Let us consider now the way in which the discretized action (19) changes under the gauge transformation

$$
\psi(x) \rightarrow e^{-i e \alpha(x)} \psi(x), \quad A_{\mu} \rightarrow A_{\mu}+\partial_{\mu} \alpha(x),
$$

which takes the discretized form

$$
\Psi \rightarrow\left(\mathbf{1}_{4} \otimes \mathbf{E}\right) \Psi, \quad \mathcal{A}_{\mu} \rightarrow \mathcal{A}_{\mu}+\Lambda_{, \mu},
$$

where $\mathbf{E}$ is the diagonal matrix whose elements are given by $\exp \left[-i e \alpha\left(x_{q}\right)\right] \delta_{q r}$ and $\Lambda_{, \mu}$ is the vector whose $q$ th component is $\partial_{\mu} \alpha\left(x_{q}\right)$, ordered according to (3). We address first our attention to the term $\bar{\Psi}\left(i \tilde{\mathbf{D}}-m \mathbf{1}_{4 N}\right) \Psi$ of $(21)$ which we denote by $\mathcal{S}_{D M}$. Under this transformation, $\mathcal{S}_{D M}$ takes the form

$$
\mathcal{S}_{D M}=\bar{\Psi}\left(\mathbf{1}_{4} \otimes \mathbf{E}^{\dagger}\right)\left[i \gamma^{\mu} \otimes\left(\mathbf{D}_{\mu}+i e \tilde{\mathcal{A}}_{\mu}+i e \tilde{\Lambda}_{, \mu}\right)-m \mathbf{1}_{4 N}\right]\left(\mathbf{1}_{4} \otimes \mathbf{E}\right) \Psi
$$

where $\tilde{\Lambda}_{, \mu}$ is the diagonal matrix whose principal diagonal is the vector $\Lambda_{, \mu}$. Therefore,

$$
\mathcal{S}_{D M}=\bar{\Psi}\left[i \gamma^{\mu} \otimes\left(\mathbf{E}^{\dagger} \mathbf{D}_{\mu} \mathbf{E}+i e \tilde{\mathcal{A}}_{\mu}+i e \tilde{\Lambda}_{, \mu}\right)-m \mathbf{1}_{4 N}\right] \Psi .
$$

In this case, (10) takes the form

$$
\mathbf{D}_{\mu} \mathbf{E}=\mathbf{E D}_{\mu}-i e \mathbf{E} \tilde{\Lambda}_{, \mu}-\boldsymbol{\Delta}_{\mu}
$$

and this gives

$$
\mathcal{S}_{D M}=\bar{\Psi}\left(i \tilde{\mathbf{D}}-m \mathbf{1}_{4 N}\right) \Psi-\bar{\Psi}\left(i \gamma^{\mu} \otimes \mathbf{E}^{\dagger} \boldsymbol{\Delta}_{\mu}\right) \Psi
$$

so that, if $N_{\mu} \rightarrow \infty$, the residual vectors vanish yielding invariance of $\mathcal{S}_{D M}$ under (31).

To show the invariance of $\mathcal{S}_{M}$, the term of (21) depending only on the gauge field, let us write the vector $\Lambda_{, \mu}$ of (31) as $\Lambda_{, \mu}=\mathbf{D}_{\mu} \Lambda+R_{\mu}$, where $\Lambda$ is the vector of values $\alpha\left(x_{q}\right)$ and $R_{\mu}$ is the residual vector. Since $\alpha(x)$ can be expanded in the basis $u_{q}(x)$ and $\mathbf{D}_{\mu}$ is an exact representation of $\partial_{\mu}$ for these functions (save for a similarity transformation), $R_{\mu}$ must vanish when $N \rightarrow \infty$, so that (31) takes the asymptotic form

$$
\Psi \rightarrow\left(\mathbf{1}_{4} \otimes \mathbf{E}\right) \Psi, \quad \mathcal{A}_{\mu} \rightarrow \mathcal{A}_{\mu}+\mathbf{D}_{\mu} \Lambda
$$

Because of $\left[\mathbf{D}_{\mu}, \mathbf{D}_{\nu}\right]=0, \mathcal{S}_{M}$ becomes invariant in such a limit and this completes the proof of gauge invariance of (21) when $N_{\mu} \rightarrow \infty$.

Now, we want to show the way in which the commutator of the discretized form of the gauge covariant derivative, Eq. (20), is related to the electromagnetic field tensor $F_{\mu \nu}$. By applying the commutator $\left[\tilde{\mathbf{D}}_{\mu}, \tilde{\mathbf{D}}_{\nu}\right]$ to $\Psi$, we get

$$
\left[\tilde{\mathbf{D}}_{\mu}, \tilde{\mathbf{D}}_{\nu}\right] \Psi=i e\left(\tilde{\mathcal{A}}_{\nu, \mu}-\tilde{\mathcal{A}}_{\mu, \nu}\right) \Psi+i e\left(\boldsymbol{\Delta}_{\nu}-\boldsymbol{\Delta}_{\mu}\right) \Psi
$$

where we have used again (10). Thus, the expression for $F_{\mu \nu}$ in terms of the commutator of the covariant derivative can be recovered when $N_{\mu} \rightarrow \infty$. 


\section{Final remarks}

Two facts about the discretization procedure presented in this letter should be noticed.

1. Since it is a nonlocal method (the matrix representations of the partial derivatives involve values of the field at each node) based on a non-equispaced lattice and the finite number of nodes breaks down the translational invariance, the NielsenNinomiya theorem does not applies in this instance. In fact, this technique yields the right results: the chiral symmetry and the dispersion relation are obtained correctly when the number of nodes $N$ is finite; the quantum commutators, the free propagators, the gauge invariance and the commutator of the covariant derivative are recovered when $N$ tends to infinity. The translational invariance is also recovered in such a limit since the nodes becomes dense in the space-time.

2. Such a technique involves matrices with a well-behaved structure. Thus, it could be a good issue to obtain analytical solutions in some cases.

\section{References}

[1] K.G. Wilson, Phys. Rev. D10 (1974) 2445.

[2] H.B. Nielsen and M. Ninomiya, Nucl. Phys. B185 (1981) 20; ibid. B193 (1981) 173.

[3] Y. Shamir, Nucl. Phys. (Proc. Suppl.) B47 (1996) 212.

[4] D.B. Kaplan, Phys. Lett. B288 (1992) 342.

[5] P.H. Ginsparg and K.G. Wilson, Phys. Rev. D25 (1982) 2649.

[6] G.T. Bodwin, Phys. Rev. D54 (1996) 6497.

[7] Pedro J. de A. Bicudo, Phys. Lett. B478 (2000) 379.

[8] Ting-Wai Chiu, hep-lat/0008010.

[9] R.G. Campos and L.O. Pimentel, Phys. Lett. A266 (2000) 98.

[10] R.G. Campos and L. Z. Juárez, Nuovo Cimento B100 (1992) 703.

[11] R.G. Campos and L.O. Pimentel, J. Comp. Phys. 160 (2000) 179.

[12] R.G. Campos and L.O. Pimentel, quant-ph/0008120 .

[13] R.G. Campos, Bol. Soc. Mat. Mexicana, 3 (1997) 279.

[14] F. Calogero, Lett. Nuovo Cimento 35 (1982) 273. 